\begin{tabular}{|c|c|c|}
\hline Beitr. Ent. & Keltern & ISSN 0005-805X \\
\hline $\mathbf{5 9}(2009) 1$ & S. $211-231$ & 15.07 .2009 \\
\hline
\end{tabular}

\title{
Vier neue Arten der Gattung Pleurocerinella BrunetTi, 1923 und Anmerkungen zu weiteren Arten der Gattung
}

\section{(Diptera: Conopidae)}

Mit 33 Figuren

Jens-Hermann Stuke

\section{Zusammenfassung}

Pleurocerinella haladai STUKe spec. nov., Pleurocerinella bartaki STUKe spec. nov. and Pleurocerinella copelandi STUKe spec. nov. werden aus dem südlichen Afrika und Pleurocerinella srilankai STUke \& Camras spec. nov. wird von Sri Lanka beschrieben. Für Pleurocerinella dioctriaeformis BrunetTi, 1923 wird ein Lectotypus designiert. Ein Bestimmungsschlüssel für die acht Arten der Gattung Pleurocerinella BRUNETTI, 1923 wird vorgelegt.

\section{Summary}

Pleurocerinella haladai STUKE spec. nov., Pleurocerinella bartaki STUKE spec. nov. and Pleurocerinella copelandi Stuke spec. nov. are described from southern Africa and Pleurocerinella srilankai Stuke \& Camras spec. nov. is described from Sri Lanka. A lectotype is designated for Pleurocerinella dioctriaeformis BRUnETTI, 1923, and a key to the eight species of the genus Pleurocerinella BrunetTi, 1923 is presented.

\section{Stichwörter}

Diptera, Conopidae, Pleurocerinella, spec. nov., Lectotypus, Bestimmungsschlüssel, Botswana, Kenia, Mozambik, Namibia, Südafrika, Tanzania, Zimbabwe, Sri Lanka.

\section{Key words}

Diptera, Conopidae, Pleurocerinella, spec. nov., lectotype, key, Botswana, Kenya, Mozambique, Namibia, South Africa, Tanzania, Zimbabwe, Sri Lanka.

\section{Neue Arten}

Pleurocerinella haladai Stuke spec. nov., Pleurocerinella bartaki Stuke spec. nov., Pleurocerinella copelandi STUKE spec. nov.

\section{Einleitung}

Die Gattung Pleurocerinella kann aufgrund der charakteristischen Fühlerform mit dem stark verlängerten Postpedicellus (Fig. 2) mit den Gattungen Euconops KRöBER, 1915, Tammo Stuke, 2008 und Jelte Stuke, 2008 verwechselt werden. Camras (2001) und Stuke (2008) stellen die Unterscheidung dieser vier Gattungen von Pleurocerinella dar. In unbestimmten Conopidenmaterial aus der Afrotropis und der Orientalis wurden mir auch verschiedene Arten aus der nur selten in Sammlungen vorkommenden Gattung Pleurocerinella BruneTTI, 1923 zur Verfügung gestellt. Dieses Material war Anlass für eine intensivere Beschäftigung mit der Gattung. Die Ergebnisse dieser Untersuchungen werden hier vorgestellt. 


\section{Methoden}

Das untersuchte Material und sein Verbleib wird bei den Artbearbeitungen aufgelistet. Die Terminologie zur Beschreibung der Morphologie entspricht McAlpine (1981) und Stuckenberg (1999). Die Homologisierung der Strukturen des Hypandriums und des Aedeagus ist derzeit nicht geklärt. Zur Untersuchung der Genitalstrukturen sowie der taxonomisch wichtigen Sternite ist es unumgänglich das gesamte Abdomen in Natronlauge $\left(\mathrm{NaOH}_{\mathrm{aq}}\right)$ zu mazerieren und anschließend zu sezieren. Bemerkenswerterweise finden sich im männlichen Genital im Gegensatz zu verschiedenen anderen Conopidengattungen bei Pleurocerinella wichtige Differentialmerkmale. Bei Holotypen wird die Originaletikettierung wiedergegeben. Dazu werden Zeilenumbrüche mit einem „/“ markiert und die einzelnen Etiketten von oben nach unten durchnummeriert.

\section{Ergebnisse}

\section{Pleurocerinella albohalterata SMITH, 1960}

(Fig. 24-25)

SмITH (1960: 43-44).

Material:

$1 \mathrm{o}^{\star}$, South Africa, Western Cape, $3 \mathrm{~km} \mathrm{~S}$ Uniondale, $610 \mathrm{~m}, 33^{\circ} 40,50^{\prime} \mathrm{S}, 23^{\circ} 08,71^{\prime} \mathrm{E}$, dry wash, hand net, 20.XI.1999, leg. M. E. Irwin, E. I. Schlinger \& F. D. Parker, coll Stuke; $1 \sigma^{*}$ South Africa, Eastern Cape, Rivierberg Range, Plessierrivier, $43 \mathrm{~km} \mathrm{NE}$ Willowmore at hwy R337, $650 \mathrm{~m}, 33^{\circ} 08,31^{\prime} \mathrm{S}, 23^{\circ} 50,40^{\prime} \mathrm{E}$, malaise trap, 18.-22.XI.1999, leg. M. E. Irwin, E. I. Schlinger \& F. D. Parker, coll. Hauser.

Von Pleurocerinella albohalterata ist bislang nur der weibliche Holotypus aus Mposa [KwaZuluNatal, Südafrika] bekannt gewesen. Bei einem überprüften Beleg des von Camras (1962) publizierten Materials vom Drachenberg [KwaZulu-Natal, Südafrika] handelt es sich um eine unbeschriebene Pleurocerinella spec. und definitiv nicht um Pleurocerinella albohalterata. Die hier präsentierten Tiere entsprechen der Originalbeschreibung bis auf die geschlechtsspezifischen Unterschiede. Der Holotypus befindet sich laut der Originalbeschreibung im „Department of Agriculture, Technical Services, Division of Entomology, Pretoria" und wurde nicht untersucht.

\section{Pleurocerinella bartaki STUKE spec. nov.}

(Fig. 1-3, 29, 33)

\section{Holotypus:}

$1 \sigma^{\star}$ aus Botswana mit folgenden Etiketten: (1) „Betschmanaland Prot. / Kalahari / Severelela - Kooa / XI.04 / L. Schultze S.“; (2) „Pleurocerinella / dioctriaeformis Brun.“ [Handschrift von Kröber] (4) „Holotypus / Pleurocerinella / bartaki / Stuke det 2009“ [Das Abdomen des Holotypus ist abgetrennt, mazeriert und befindet sich in einem mit Glyzerin gefüllten Plastikrohr am Tier. Ansonsten fehlen dem Tier die mittleren Beine, die Hintertarsen, die linke Flügelspitze und die rechte Haltere.]

\section{Paratypus:}

Mosambik: $10^{\star}$, 35km SW Chimoio, 19 008'S, 3309'E, 700m, 14.XII.2005, leg. Halada, coll. Stuke. 

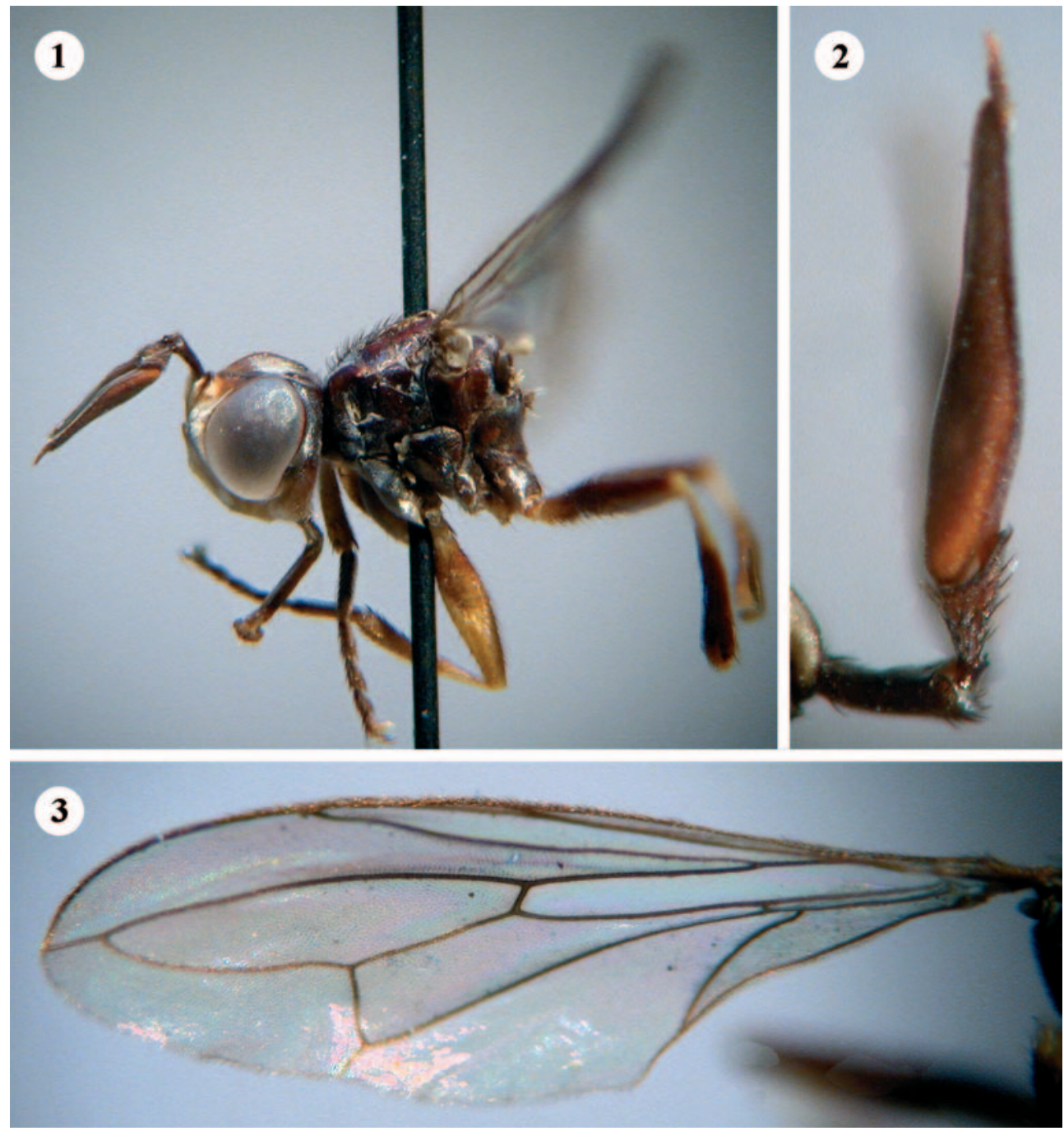

Fig. 1-3: Pleurocerinella bartaki spec. nov. (Holotypus): 1 Habitus; 2 Fühler; 3 Flügel.

Der Holotypus befindet sich in der Sammlung des Museums für Naturkunde der HumboldtUniversität (ZMHB). Der Paratypus befindet sich in der Privatsammlung Stuke.

\section{Beschreibung des Holotypus $\left(0^{\top}\right)$ :}

Gesamtlänge 6,4 mm (Abdomenspitze bis Stirn, ohne Fühlerlänge).

\section{Kopf:}

Kopfhöhe 1,2 mm (maximale Länge Augenoberrand bis Kopfunterrand). Auge unbehaart, hellbraun. Ocellen braun, auf einem schwach bestäubten braunen Ocellenhöcker gut sichtbar. Fühler einheitlich hellbraun, Pedicellus vorne an der Unterseite gelbbraun. Außenseite des Scapus und gesamter Pedicellus mit schwarzen Haaren. Stylus dreigliedrig, mittleres Glied deutlich nach unten ausgezogen. Form der Fühlerglieder entsprechend Figur 2. Stirn braun, anterior gelbbraun 
werdend, unbehaart, am Hinterrand mit silberner Bestäubung. Vertex glänzend braun, ohne auffällige Haare. Occiput braun, in der unteren Hälfte gelbbraun. Occiput mit silberner Bestäubung und schwarzen bis braunen Haaren. Parafacia schmaler als Postpedicellus, gelb, silbern bestäubt, mit einzelnen schwarzen Haaren am Übergang zur Fühlergrube. Fühlergruben und Mittelkiel mit Clypeus gelb, silbern bestäubt. Genae gelb, nur schwach bestäubt, mit einzelnen braunen Haaren. Rüssel braun, Labellum erweitert, rudimentäre Palpen braun.

\section{Thorax:}

Thorax braun. Mesoscutum, Postpronotum (Humerus) und Scutellum mit schwarzen Haaren. 3-4 Borsten am Propleuron oberhalb der Vordercoxen, 1 Borste am dorsalen Hinterrand des Katepisternums. Pleuren weiß bestäubt, nur das Katepisternum in der Mitte glänzend. Mesoscutum nur lateral und vorne bestäubt, in der Mitte glänzend.

Coxen hellbraun, mit einzelnen schwarzen Haaren und dicht silbern bestäubt. Trochanter gelbbraun, unbestäubt, ebenfalls mit einzelnen schwarzen Haaren. Hinterfemur an Basis und an Spitze schmal gelbbraun, ansonsten braun. Vorderfemur heller gelbbraun. Hintertibie basal zu einem Drittel blassgelb, ansonsten braun. Vordertibie gelbbraun. Tarsen gelbbraun, durch die dichte schwarze Behaarung dunkler wirkend. Klauen, Pulvillen und Empodien hellgelb bis auf schwarzes apikales Drittel der Klauen. Beine schwarz behaart. Femora an der Unterseite apikal mit einzelnen schwarzen Borsten. Apikale Hälfte der Hintertibie ventral genauso wie vorhandene Basitarsen mit dichter hellgelber Behaarung.

Flügellänge 4,4 mm (gemessen von Flügelspitze bis Tegula). Flügel vollstänfig hyalin. Flügel bis auf die gesamte vorderen Basalzelle (br), die gesamte hintere Basalzelle (bm), die basale Costalzelle (bc), die Basis der Subcostalzelle (sc) und die Basis der Radialzelle (r1) vollständig mit Mikrotrichen besetzt. Flügeladerung entsprechend Fig. 3. Haltere gelbweiss, basal hellbraun. Sowohl am Capitulum als auch am Pedicellus mit einzelnen schwarzen Borsten. Obere und untere Calyptra gelbweiss, obere Calyptra am Rand mit hellen Härchen besetzt.

\section{Abdomen:}

Abdomen braun. Bestäubung kann am mazerierten Tier nicht nachgewiesen werden. Tergite und Sternite vollständig mit schwarzen Haaren besetzt. Sternit 5 am Hinterrand mit breitem schwarzem Borstenfeld. Epandrien distal vor den Cerci verschmolzen, dieser Bereich ist aber weniger stark sklerotisiert (Fig. 33). Unpaarer Zahn an Aedeagus entsprechend Fig. 29.

\section{Beschreibung ㅇ:}

Das Weibchen dieser Art ist unbekannt geblieben.

\section{Variationsbreite:}

Flügellänge des Paratypus 5,5 mm. Der Paratypus ist insgesamt schwarz bis schwarzbraun, die braune Grundfärbung beim Holotypus könnte eine Folge des Alters sein. Die Augen des Paratypus sind rotbraun. Der Vertex ist beim Paratypus bestäubt und es finden sich lange schwarze Haare. Der Mittelfemur ist wie der Vorderfemur gefärbt.

\section{Derivatio nominis:}

Die Art wird nach Prof. Dr. Miroslav Barták (Prag) benannt, von dem ich außerordentlich umfangreiches Conopidenmaterial zur Bearbeitung zugesandt bekommen habe. Unter anderem auch Material von zwei der hier neu beschriebenen Arten. Darüber hinaus hat sich Miroslav Barák mit seiner immensen Sammeltätigkeit in hohem Maße um die Erforschung der Conopidenfauna der Tschechischen Republik und der Slowakei verdient gemacht. 


\section{Diagnose:}

Die Bestimmung von Pleurocerinella bartaki ist mit dem unten beigefügten Bestimmungsschlüssel möglich. Pleurocerinella bartaki unterscheidet sich von den afrotropischen Pleurocerinella-Arten durch folgende Merkmalskombination: (1) Mesoscutum medial glänzend, (2) Hinterfemur verdunkelt (braun bis schwarz), (3) Femora mit einzelnen schwarzen Borsten (4) Fehlen von Mikrotrichen in den Basalzellen br und bm und der Basis von der Radialzelle r1 und (5) das vor den Cerci verschmolzene Epandrium (Fig. 33).

\section{Diagnosis:}

The identification characteristics of this species are set out in the key below. Pleurocerinella bartaki differs from the other afrotropical Pleurocerinella through the following combination of characteristics: (1) mesoscutum undusted medially; (2) hind femur darkened (brown - black); (3) femora ventrally with single black spine at apex; (4) the lack of microtrichia in the cells br and bm, and at the base of cell $r 1$; and (5) the complete fusion of the epandrium margins distal to the cerci (Fig. 33).

\section{Verbreitung:}

Pleurocerinella bartaki ist nach dem hier untersuchten Material aus Botswana und Mosambik bekannt geworden. In Mosambik flog die Art zusammen mit Pleurocerinella haladai.

\section{Pleurocerinella copelandi STUKE spec. nov.}

(Fig. 4-7, 30-31)

\section{Holotypus:}

$1 \sigma^{\star}$ aus Botswana mit folgenden Etiketten: (1) „BOTSWANA: Serowe / Farmer's Bridge / Forchhammer

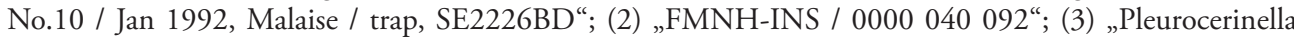
/ albohalterata / Smith / det. Camras“; (4) „Holotypus / Pleurocerinella / copelandi / Stuke det 2009“ [Das Abdomen des Holotypus ist abgetrennt, mazeriert und befindet sich in einem mit Glyzerin gefüllten Plastikröhrchen am Tier. Ansonsten ist das Tier vollständig und abgesehen von den typischen Effekten durch das Trocknen von Alkoholmaterial gut erhalten.]

\section{Paratypus:}

Namibia:1 ơ, Otavi, 25.XII.1932, leg. L. Ogilvie, coll. BMNH [det. Kröber, 1938, als „Pleurocerinella dioctriaeformis Brun."].

Der Holotypus befindet sich in der Sammlung Camras, die in die Sammlung des Field Museum of Natural History, Chicago (FMNH) integriert werden wird. Der Paratypus befindet sich in der Sammlung des Natural History Museum [früher British Museum (Natural History)] (BMNH).

\section{Beschreibung des Holotypus $\left(\sigma^{\top}\right)$ :}

Gesamtlänge 5,1 mm (Abdomenspitze bis Stirn, ohne Fühlerlänge).

\section{Kopf:}

Kopfhöhe etwa 0,9 mm (maximale Länge Augenoberrand bis Kopfunterrand, Ungenauigkeiten ergeben sich, da der Kopf beim Trocknen etwas eingefallen ist). Auge unbehaart, rotbraun. Ocellen orangebraun, auf einem schwach bestäubten schwarzen Ocellenhöcker gut sichtbar. Scapus und Pedicellus schwarzbraun, Postpedicellus dunkelbraun und ventral aufgehellt. Außenseite des Scapus und gesamter Pedicellus mit schwarzen Haaren. Stylus zweigliedrig, basales Glied deutlich 

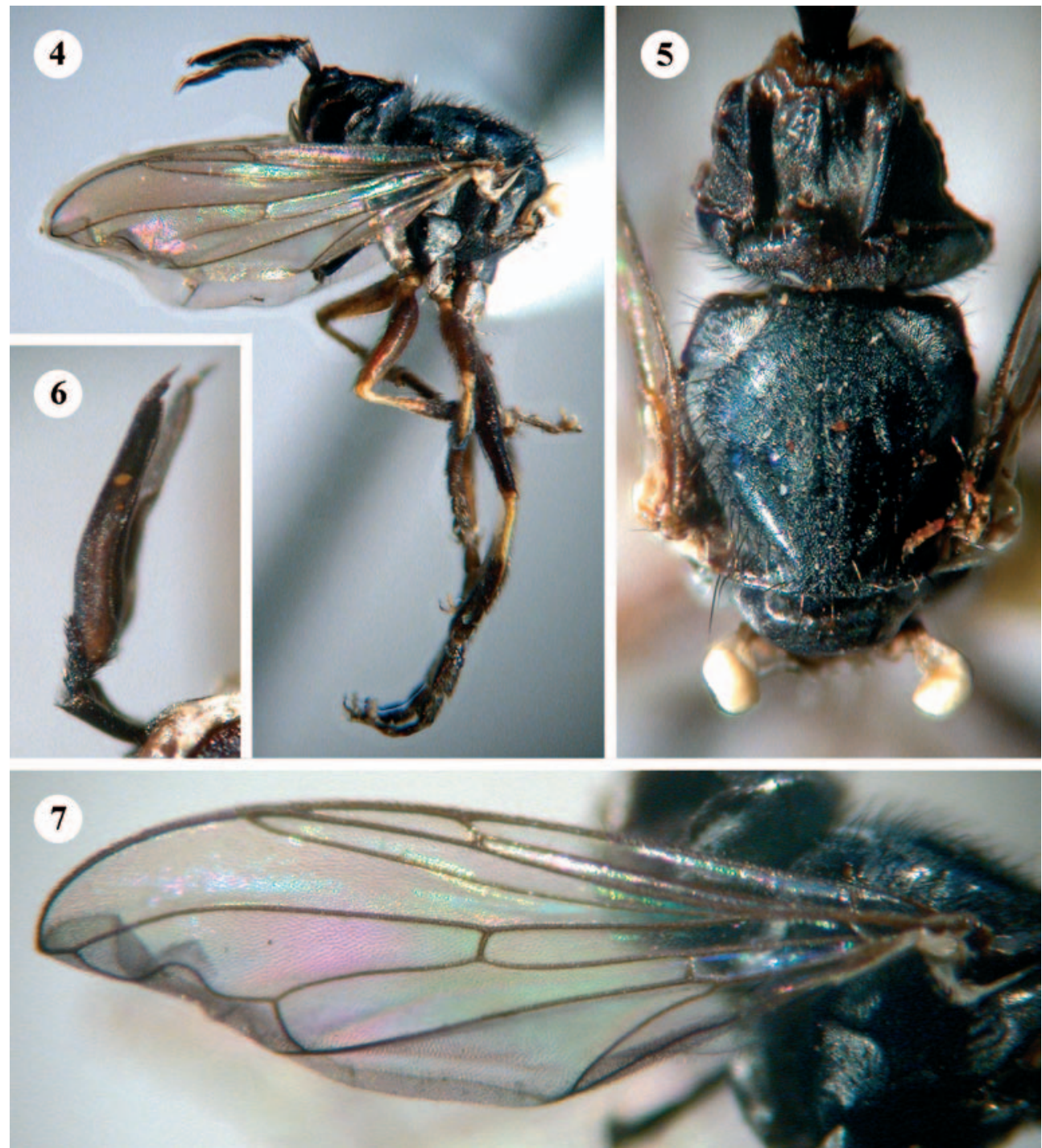

Fig. 4-7: Pleurocerinella copelandi spec. nov. (Holotypus): 4 Habitus; 5 Mesoscutum; 6 Fühler; 7 Flügel.

nach unten ausgezogen. Eventuell kann das zu erwartende dritte Stylusglied bei dem getrockneten Tier nicht mehr erkannt werden. Form der Fühlerglieder entsprechend Fig. 6. Stirn dunkelbraun und apikal gelbbraun werdend, unbehaart, am Hinterrand mit silberner Bestäubung. Vertex glänzend schwarzbraun mit langen schwarzen Haaren. Occiput schwarzbraun, ventral aufgehellt gelbbraun. Occiput mit dichter silberner Bestäubung und einzelnen schwarzen Haaren. Parafacia schmaler als Postpedicellus, gelb, silbern bestäubt, mit einzelnen schwarzen Haaren am Übergang zur Fühlergrube. Fühlergruben und Mittelkiel mit Clypeus gelb, dicht silbern bestäubt. Genae gelb, nur schwach bestäubt. Rüssel schwarz, Labellum erweitert (Fig. 3), rudimentäre Palpen braun. 


\section{Thorax:}

Thorax schwarz. Mesoscutum, Postpronotum (Humerus) und Scutellum mit schwarzen Haaren. 2 Borsten am Propleuron oberhalb der Vordercoxen, 2 Borsten am dorsalen Hinterrand des Katepisternums. Pleuren weiß bestäubt, nur das Katepisternum in der Mitte glänzend. Mesoscutum bis auf submediale Streifen deutlich bestäubt (Fig. 5).

Vordercoxe gelbbraun, Mittel- und Hintercoxen schwarzbraun. Alle Coxen mit einzelnen schwarzen Haaren und dicht silbern bestäubt. Trochanter gelbbraun, unbestäubt, ebenfalls mit einzelnen schwarzen Haaren. Hinterfemur an Basis und an Spitze schmal gelbbraun, ansonsten schwarzbraun. Mittel- und Vorderfemur heller gelbbraun. Hintertibie basal zu einem Drittel blassgelb, ansonsten schwarz. Vorder- und Mitteltibie blassgelb, im apikalen Drittel schwach verdunkelt. Tarsen gelbbraun, durch die dichte schwarze Behaarung dunkler wirkend. Klauen, Pulvillen und Empodien hellgelb bis auf schwarzes apikales Drittel der Klauen. Beine schwarz behaart, ohne schwarze Borsten an der Unterseite der Femora. Apikale Hälfte der Hintertibien ventral genauso wie alle Basitarsen mit dichter hellgelber Behaarung.

Flügellänge 3,5 mm (gemessen von Flügelspitze bis Tegula). Flügel vollständig hyalin. Flügel bis auf die Basis der vorderen Basalzelle (br), die hintere Basalzelle (bm), die basale Costalzelle (bc), die Basis der Subcostalzelle (sc) und die Basis der Radialzelle (r1) vollständig mit Mikrotrichen besetzt. Flügeladerung entsprechend Figur 7. Haltere gelbweiss, basal hellbraun. Sowohl am Capitulum als auch am Pedicellus einzelne schwarzen Borsten. Obere und untere Calyptra gelbweiss, obere Calyptra am Rand mit hellen Härchen besetzt.

\section{Abdomen:}

Abdomen schwarzbraun. Bestäubung kann am mazerierten Tier nicht nachgewiesen werden. Tergite und Sternite vollständig mit schwarzen Haaren besetzt. Sternit 5 am Hinterrand mit breitem schwarzem Borstenfeld. Epandrien distal vor den Cerci verschmolzen (Fig. 31). Unpaarer Zahn an Aedeagus entsprechend Figur 30.

\section{Beschreibung 9 :}

Das Weibchen dieser Art ist unbekannt geblieben.

\section{Variationsbreite:}

Bei dem Paratypus ist der Vorderfemur geringfügig stärker verdunkelt. Die Flügel sind $4 \mathrm{~mm}$ lang.

\section{Derivatio nominis:}

Die Art wird nach dem Sammler Robert Copeland benannt. Der Autor ist außerordentlich dankbar für das vielfältige Conopiden-Material, das Robert Copeland aus Afrika gesammelt hat und zur Bearbeitung zur Verfügung gestellt hat. Unter anderem befanden sich darunter auch zwei Weibchen von Pleurocerinella, die zu Pleurocerinella copelandi gehören könnten.

\section{Diagnose:}

Die Bestimmung der Art ist mit dem unten beigefügten Bestimmungsschlüssel möglich. Pleurocerinella copelandi ist besonders durch das medial stark bestäubte Mesoscutum charakerisiert (Fig. 5) und kann durch dieses Merkmal von allen anderen bekannt gewordenen afrotropischen Pleurocerinella-Arten unterschieden werden. 


\section{Diagnosis:}

The identification characteristics of this species are set out in the key below. Pleurocerinella copelandi is characterised by having the mesoscutum strongly dusted medially and can be differentiated by this character from all other afrotropical Pleurocerinella species.

\section{Verbreitung:}

Pleurocerinella copelandi kommt nach dem hier untersuchten Material in Botswana und Namibia vor.

\section{Pleurocerinella dioctriaeformis BRUNETTI 1923}

(Fig. 21, 23, 26)

Brunetti (1923: 368-369).

\section{Lectotypus:}

$1 \sigma^{\top}$ aus Indien mit folgenden Etiketten: (1) „SYNTYPE“ [rundes Etikett mit blauem Rand]; (2) „Simla / 8.98.“; (3) „Syntype ơ of / Pleurocerinella / dioctriaeformis / Brunetti, 1923 / India: Simia / coll. C. G. Nurse“; (4) „Lectotypus / Pleurocerinella / dioctriaeformis / Brunetti, 1923 / Stuke des. 2008“. [Der Lectotypus befindet sich in der Sammlung des Natural History Museum [früher British Museum (Natural History)] (BMNH). Außer dem fehlenden rechten Hinterbein und fehlenden Fühlern ist er in gutem Zustand.]

Weiteres Material:

China: 1 ๙ $^{\star}$, „Charbin“ (= Harbin), 02.VIII.1938, coll. Deutsches Entomologisches Institut, Müncheberg (DEI); - Nepal: 1 + , Ktmd., Godavari, 5000', 03.-07.VIII.1967, Can. Nepal, Exped., coll. Camras; 1 ơ, Ktmd., Godavari, 5000', R. Bot. Gdn. 17.VII.1967, Can. Nepal, Exped., coll. Camras; 1 ơ $^{\star}$ Ktmd., Godavari, 6000', 20.-22.VII.1967, Malaise trap, Can. Exp., coll. Camras.

Es wurde nur ein Tier aus der Syntypenserie von insgesamt drei Tieren ausgeliehen. Um den Gebrauch des Namens zu stabilisieren, wird dieses Exemplar als Lectotypus designiert.

Beschrieben wurde die Art von Simla (= Shimla [Indien]) und Maskeliya [Sri Lanka]. Der Paralectoypus aus Sri Lanka wurde nicht untersucht und es ist nicht auszuschließen, dass es sich dabei um die hier neu beschriebene und ähnliche Pleurocerinella srilankai handelt. Bei Meldungen für Afrika (BRUnetTi 1925, Sмith 1980) ist es wahrscheinlich, dass es sich um Verwechselungen mit einer der in dieser Arbeit beschriebenen afrotropischen Pleurocerinella handelt. Es liegen als Pleurocerinella dioctriaeformis fehlbestimmt Tiere von Pleurocerinella haladai und Pleurocerinella bartaki vor. Demnach wäre Pleurocerinella dioctriaeformis nach geprüften Belegen nur aus dem nördlichen Indien, Nepal und Nordchina bekannt.

\section{Pleurocerinella haladai STUKE spec. nov.}

(Fig. 8-14, 27, 32)

\section{Holotypus:}

$1 \mathrm{o}^{\top}$ aus Mozambik mit folgenden Etiketten: (1) „Mozambik -CW / 35km SW Chimoio / S 190 $08^{\prime}$ E $33^{\circ} 09^{\prime}$ / 700m 14.12.2005 / Kadlecová leg.“; (2) „Holotypus / Pleurocerinella haladai / spec. nov. o / Stuke det. 2008" [Der Holotypus ist komplett und in gutem Zustand.] 


\section{Paratypen:}

Mozambik: $50^{\star} 0^{\star}, 1$ 온, Daten wie Holotypus, leg. Kadlecová \& Hallada, coll. Bartak \& Stuke; - Zimbabwe: Umtali [= Mutare], II.1917, coll. BMNH [det. Brunetti, 1924, als „Pleurocerinella dioctriaeformis Brun. ơ"].

Der Holotypus wird in der Sammlung des Deutschen Entomologischen Instituts (Müncheberg) aufbewahrt. Paratypen befinden sich in der Sammlung des Natural History Museum [früher British Museum (Natural History)] (BMNH) und den Privatsammlungen von Barták und Stuke.

\section{Beschreibung des Holotypus $\left(0^{\star}\right)$ :}

Gesamtlänge 8,5 mm (Abdomenspitze bis Stirn, ohne Fühlerlänge).

\section{Kopf:}

Kopfhöhe 1,5 mm (maximale Länge Augenoberrand bis Kopfunterrand). Auge unbehaart, rotbraun, Facetten im vorderen Augenbereich größer. Ocellen orangebraun, auf einem glänzenden schwarzen Ocellenhöcker gut sichtbar. Scapus und Pedicellus schwarz, Postpedicellus dunkelbraun und ventral aufgehellt. Außenseite des Scapus und gesamter Pedicellus mit schwarzen Haaren. Stylus dreigliedrig, mittleres Glied deutlich nach unten ausgezogen. Form der Fühlerglieder entsprechend Figur 2. Stirn schwarz, unbehaart, am Hinterrand mit silberner Bestäubung. Vertex glänzend schwarz mit langen schwarzen Haaren. Occiput schwarz mit dichter silberner Bestäubung und einzelnen schwarzen Haaren. Parafacia schmaler als Postpedicellus, gelb, silbern bestäubt, mit einzelnen schwarzen Haaren am Übergang zur Fühlergrube. Fühlergruben im oberen Bereich dunkelbraun, ansonsten gelb, dicht silbern bestäubt. Mittelkiel mit Clypeus gelb. Genae gelb, nur schwach bestäubt. Rüssel schwarz, Labellum erweitert (Fig. 10).

\section{Thorax:}

Thorax schwarz. Mesoscutum, Postpronotum (Humerus) und Scutellum mit schwarzen Haaren. 2 Borsten am Propleuron oberhalb der Vordercoxen, mehrere Borsten am dorsalen Hinterrand des Katepisternums. Pleuren mit weißen Mikrotrichen besetzt, die aber bis auf einen breiten Streifen von den Mittelcoxen bis kurz vor die Flügelbasis (Schillerstreifen, Fig. 11) unauffällig bleiben. Mesoscutum nur lateral schwach bestäubt.

Coxen schwarzbraun, mit einzelnen schwarzen Haaren; Vordercoxe vollständig dicht bestäubt, Mittel- und Hintercoxe nur lateral. Trochanter gelbbraun, unbestäubt, ebenfalls mit einzelnen schwarzen Haaren. Femora an Basis und an Spitze schmal gelbbraun, Hinterfemur ansonsten schwarz, Mittel- und Vorderfemur ansonsten braun. Hintertibie basal zu einem Drittel blassgelb, ansonsten schwarz. Vorder- und Mitteltibie blassgelb mit schwarzen Flecken im apikalen Drittel. Tarsen gelbbraun, durch die dichte schwarze Behaarung dunkler wirkend. Klauen, Pulvillen und Empodien hellgelb bis auf schwarzes apikales Drittel der Klauen. Beine schwarz behaart. An den apikalen Unterseiten der Femora jeweils an der Vorder- und Rückseite eine Reihe einzelner kräftiger Borsten. Apikale Hälfte der Hintertibien ventral genauso wie alle Basitarsen mit dichter hellgelber Behaarung.

Flügellänge $5,5 \mathrm{~mm}$ (gemessen von Flügelspitze bis Tegula). Flügel bis auf die vordere Basalzelle (br), die hintere Basalzelle (bm), die basale Costalzelle (bc), die Basis der Subcostalzelle (sc) und die Basis der Radialzelle (r1) vollständig mit Mikrotrichen besetzt. Flügeladerung und Färbung entsprechend Figur 12. Haltere gelbweiss, basal hellbraun. Sowohl am Capitulum als auch am Pedicellus kleine Felder mit einzelnen schwarzen Borsten. Obere und untere Calyptra gelbweiss, obere Calyptra am Rand mit hellen Härchen besetzt. 


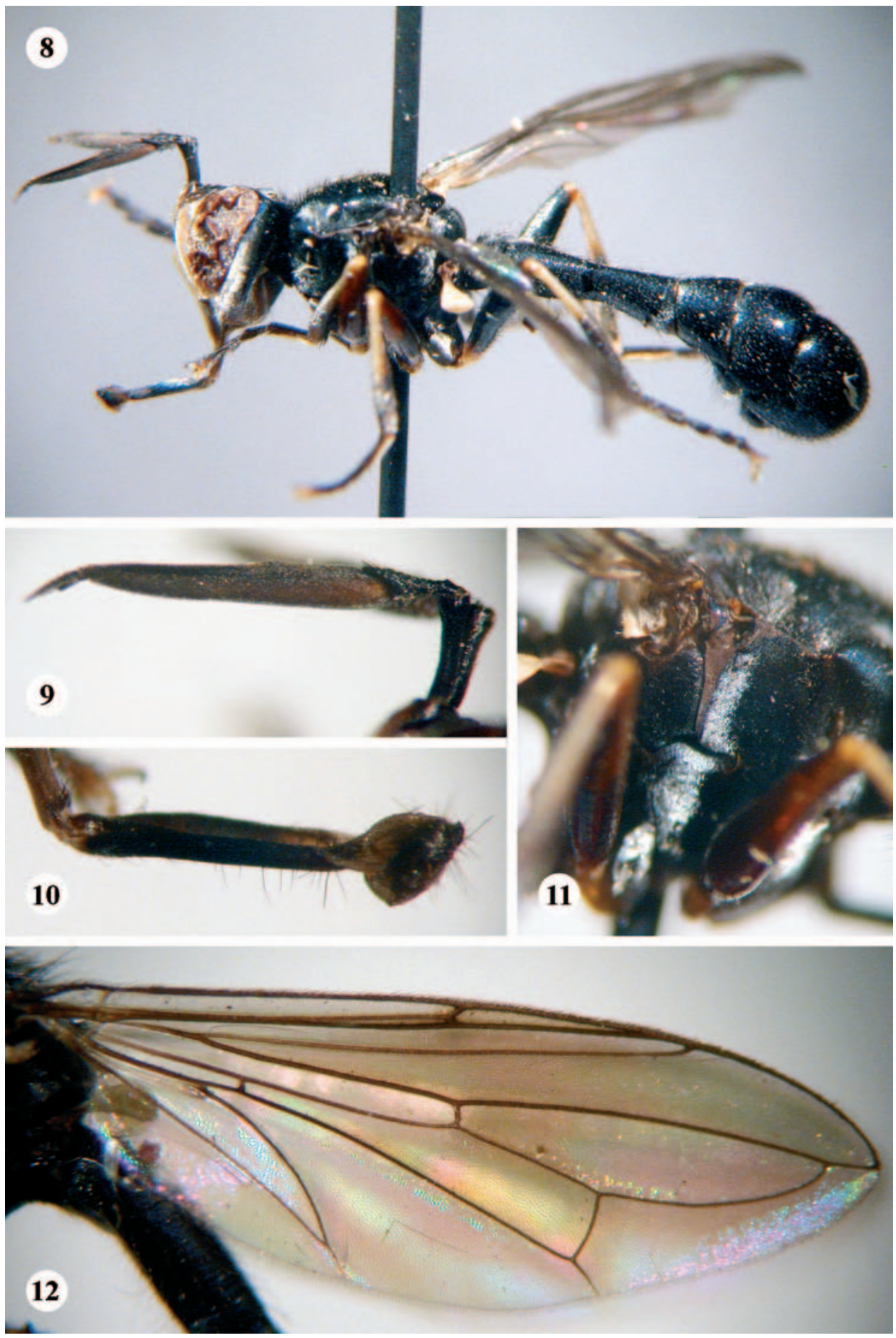

Fig. 8-12: Pleurocerinella haladai spec. nov.: 8 Habitus (Holotypus); 9 Fühler (Holotypus); 10 Labellum (Holotypus); 11 Pleuren mit Bestäubungsstreifen (Holotypus); 12 Flügel (Paratypus aus Umtali). 

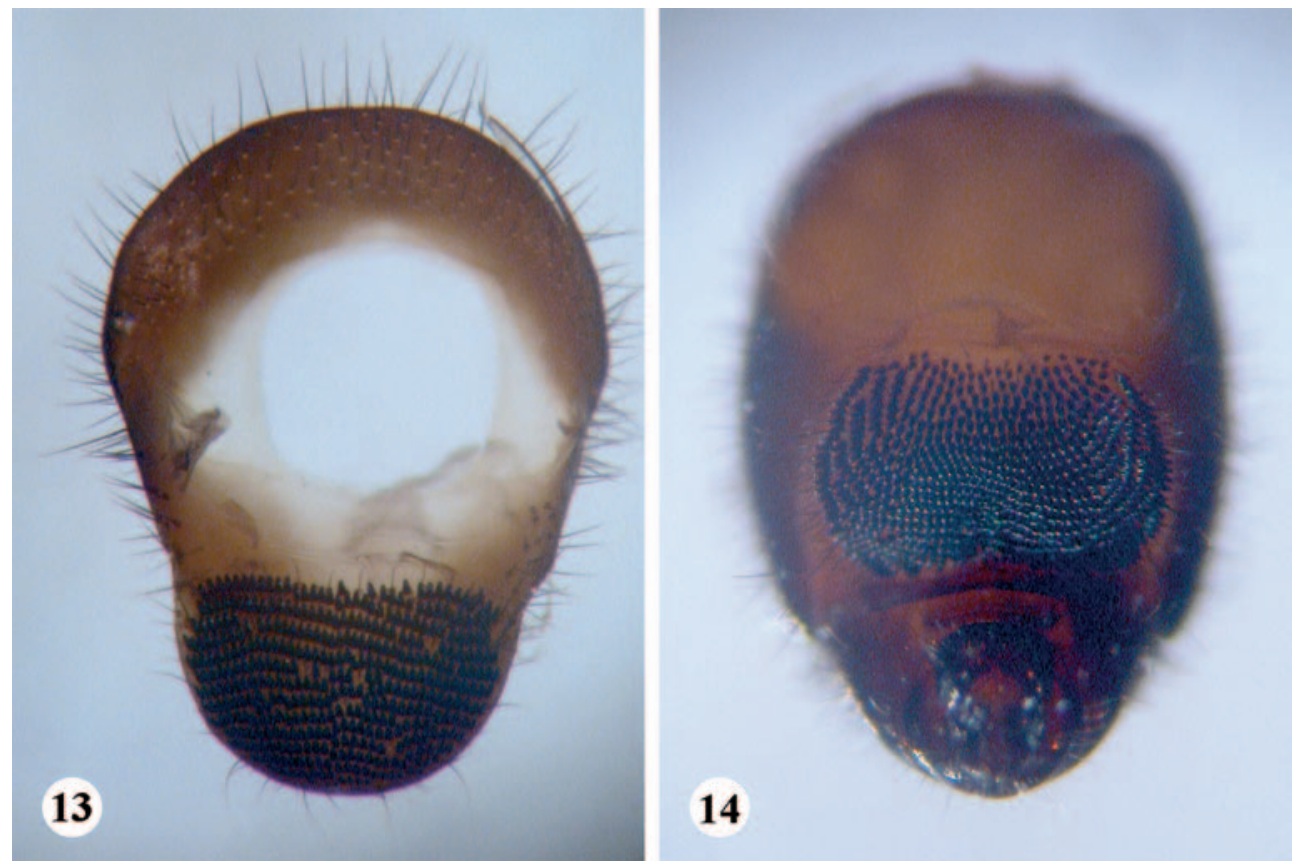

Fig. 13-14: ㅇ von Pleurocerinella haladai spec. nov. (Paratypus vom Locus typicus): 13 Theka; 14 Sternit 6 ventral.

\section{Abdomen:}

Abdomen schwarz. Unbestäubt bis auf schmale Flecken lateral an den Hinterrändern von Tergit 1-3 und das ganze Sternit 1. Tergite und soweit erkennbar auch Sternite vollständig mit schwarzen Haaren besetzt. Sternit 5 am Hinterrand mit breitem schwarzem Borstenfeld. Form des Abdomen entsprechend Figur 1. Die Genitalien des Holotypus wurden nicht untersucht.

\section{Beschreibung 우}

Das Weibchen ähnelt dem Männchen mit Ausnahme der geschlechtsspezifischen Ausbildung der Abdomenspitze. Die Theka ist in Figur 13 dargestellt, Sternit 6 in Figur 14.

\section{Variationsbreite:}

Der Pedicellus ist nicht immer ganz schwarz, sondern kann basal aufgehellt sein. Die Färbung der Mittel- und Vorderbeine ist variabel und kann überwiegend dunkelbraun bis gelb sein. Die Flügellänge variiert von 4,8-7,1 mm. Die $\sigma^{\star}$-Genitalien wurden bei zwei Paratypen untersucht: Die Epandrien sind distal vor den Cerci getrennt. Der unpaare Zahn an der Aedeagusspitze entspricht Figur 27.

\section{Derivatio nominis:}

Die Art wird nach dem Sammler der Typenserie Jiří Halada (Prag) benannt. Der Autor ist außerordentlich dankbar für das vielfältige Conopiden-Material, das Jiř́ Halada aus verschiedenen Regionen der Welt gesammelt hat. 


\section{Diagnose:}

Die Bestimmung der Art ist mit dem unten beigefügten Bestimmungsschlüssel möglich. Die Männchen von Pleurocerinella haladai unterscheiden sich leicht von allen anderen afrotropischen Pleurocerinella an dem vor den Cerci getrennten Epandrium (Fig. 32).

\section{Diagnosis:}

The identification characteristics of this species are set out in the key below. Pleurocerinella haladai differs from all other afrotropical Pleurocerinella in the male by having the epandrium margins separated distal to the cerci (Fig. 32).

\section{Verbreitung:}

Pleurocerinella haladai kommt nach bisherigem Kenntnisstand in Mozambik und Zimbabwe vor.

\section{Pleurocerinella srilankai STUKe \& CAMRAS spec. nov.}

(Fig. 15-19, 28)

\section{Holotypus:}

$10^{\top}$ aus Sri Lanka mit folgenden Etiketten: (1) „SRI LANKA:N. E. Dist. / Hankgala Sanctuary / 23-24IV-1981“; (2) „K. V. Krombein / T. Wijesinhe / L. Weeratunge“; (3) „FMNH-INS / 0000040 088“; (4) „HOLOTYPUS / Pleurocerinella srilankai spec. nov. o / det. Stuke 2008“. [Das abgetrennte Abdomen befindet sich in einer Genitalkapsel in Glycerin am Tier. Das Genital wurde mit $\mathrm{NaOH}_{(\text {aq) }}$ mazeriert.]

Der Holotypus befindet sich in der Sammlung Camras, die in die Sammlung des Field Museum of Natural History, Chicago (FMNH) integriert werden wird.

\section{Beschreibung des Holotypus ( $\left.0^{\top}\right)$ :}

Gesamtlänge etwa $8 \mathrm{~mm}$ (Abdomenspitze bis Stirn, ohne Fühlerlänge).

\section{Kopf:}

Kopfhöhe 1,7 mm (maximale Länge Augenoberrand bis Kopfunterrand). Auge unbehaart, rotbraun, Facetten im vorderen Augenbereich etwas größer. Ocellen orangebraun, auf dem schwarzen Ocellenhöcker gut sichtbar. Fühler schwarz, nur ventral auf allen drei Gliedern undeutlich braun aufgehellt. Außenseite des Scapus und gesamter Pedicellus mit schwarzen Haaren. Stylus dreigliedrig, mittleres Glied deutlich nach unten ausgezogen. Form der Fühlerglieder entsprechend Figur 17. Stirn schwarz, unbehaart, unbestäubt. Vertex glänzend schwarz mit langen schwarzen Haaren. Occiput schwarz, nur am Augenhinterrand dicht silbern bestäubt und ansonsten glänzend, mit schwarzen Haaren. Parafacia schmaler als Postpedicellus, gelb, silbern bestäubt, mit einzelnen schwarzen Haaren am Übergang zur Fühlergrube. Fühlergrube im oberen Bereich dunkelbraun, ansonsten gelb, dicht silbern bestäubt. Mittelkiel gelbbraun, dorsal scharf abgegrenzt schwarzbraun, Clypeus ebenfalls schwarzbraun. Genae gelb, nur schwach bestäubt. Rüssel braun, Labellum erweitert.

\section{Thorax:}

Thorax schwarz. Mesoscutum, Postpronotum (Humerus) und Scutellum mit schwarzen Haaren. 3 Borsten am Propleuron oberhalb der Vordercoxen, mehrere Borsten am dorsalen Hinterrand des Katepisternums. Pleuren mit ausgedehnter unauffälliger weißer Bestäubung. Unbestäubt bleiben je- 

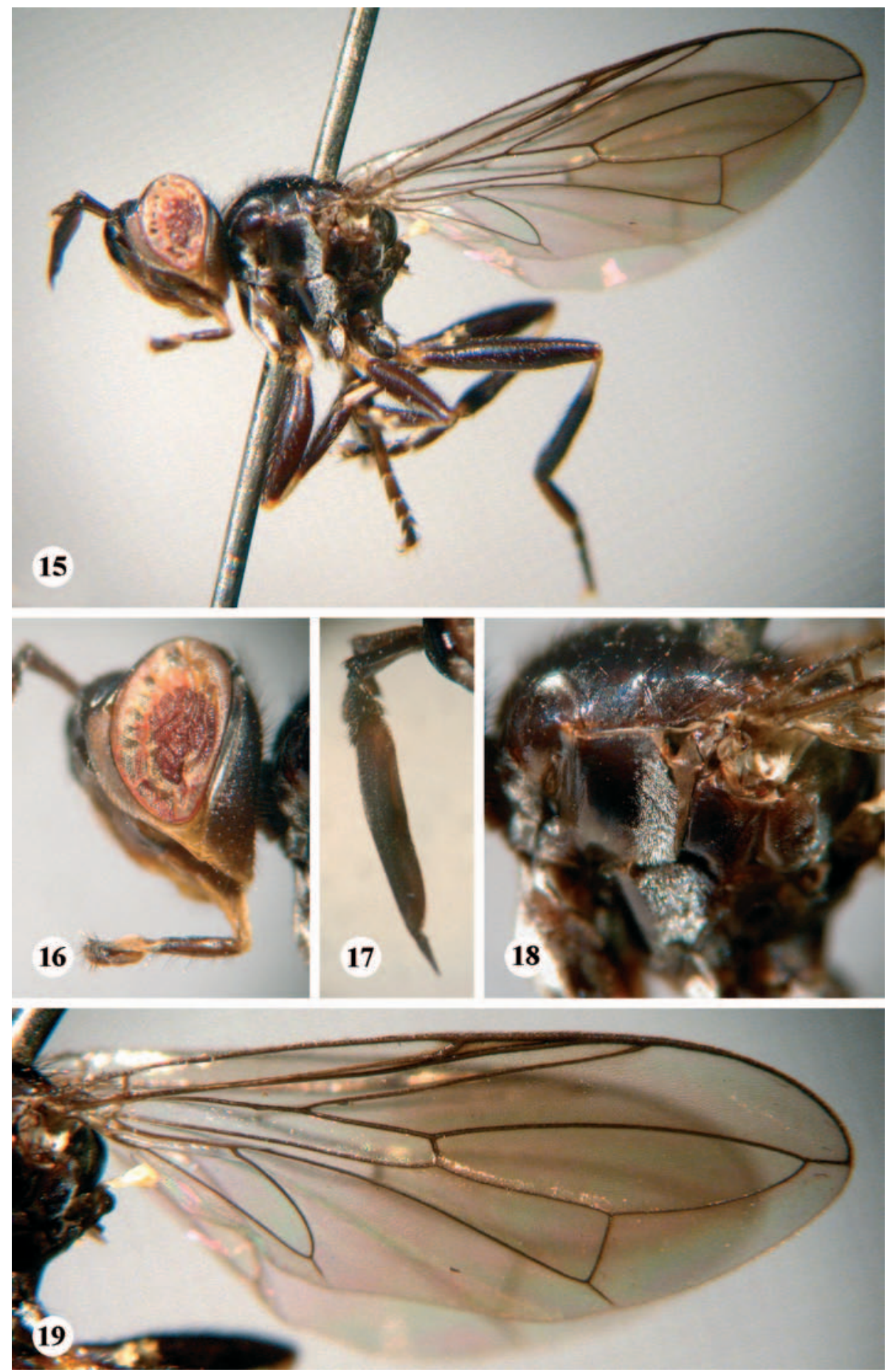

Fig. 15-19: Pleurocerinella srilankai spec. nov. (Holotypus): 15 Habitus; 16 Kopf; 17 Fühler; 18 Pleuren mit Bestäubungsstreifen; 19 Flügel. 
weils die vorderen Bereiche des Postpronotums, Anepisternums, Anepimerons, Laterotergits und Katepisternums. Ein dicht bestäubter Bereich, erstreckt sich von den Mittelcoxen bis kurz vor die Flügelbasis (Schillerstreifen, Fig. 18). Mesoscutum nur lateral bestäubt.

Coxen braun, mit einzelnen schwarzen Haaren; Vordercoxe vollständig dicht bestäubt, Mittelund Hintercoxe nur lateral. Trochanter gelbbraun, unbestäubt, ebenfalls mit einzelnen schwarzen Haaren. Femora an Basis und an Spitze sehr schmal gelb, ansonsten dunkelbraun. Tibien basal zu einem Drittel blassgelb, ansonsten dunkelbraun. Tarsen bis auf dunkelbraune Oberseiten gelbbraun, durch dichte schwarze Behaarung dunkler wirkend. Klauen, Pulvillen und Empodien hellgelb bis auf schwarzes apikales Drittel der Klauen. Beine schwarz behaart. An den apikalen Unterseiten der Femora jeweils an der Vorder- und Rückseiteseite eine Reihe einzelner kräftiger Borsten. Apikale Hälfte der Hintertibie ventral genauso wie alle Basitarsen mit dichter hellgelber Behaarung.

Flügellänge $6,7 \mathrm{~mm}$ (gemessen von Flügelspitze bis Tegula). Flügel bis auf die vordere Basalzelle (br), die hintere Basalzelle (bm), die Basis der Costalzelle (c), die basale Costalzelle (bc), die Basis der Subcostalzelle (sc) und die Basis der Radialzelle r1 vollständig mit Mikrotrichen besetzt. Flügeladerung und Färbung entsprechend Figur 19. Haltere gelbweiss, basal hellbraun. Sowohl am Capitulum als auch am Pedicellus kleine Felder mit einzelnen schwarzen Borsten. Obere und untere Calyptra gelbweiss, obere Calyptra am Rand mit hellen Härchen besetzt.

\section{Abdomen:}

Abdomen schwarz. Unbestäubt bis auf schmale Flecken lateral an den Hinterrändern von Tergit 3 und die Seiten von Sternit 1. Tergite und soweit erkennbar auch Sternite vollständig mit schwarzen Haaren besetzt. Sternit 5 am Hinterrand mit breitem schwarzem Borstenfeld. Epandrien distal vor den Cerci vollständig verschmolzen. Unpaarer Zahn an Aedeagusspitze fehlt (Fig. 28).

\section{Beschreibung 우 :}

Das Weibchen ist bislang nicht bekannt geworden.

\section{Derivatio nominis:}

Bisher ist die Art nur von Sri Lanka bekannt, auf die Verbreitung nimmt der Name Bezug.

\section{Diagnose:}

Die Bestimmung der Art ist mit dem unten beigefügten Bestimmungsschlüssel möglich. Als einzige weitere Pleurocerinella-Art im Verbreitungsgebiet von Pleurocerinella srilankai wurde Pleurocerinella dioctriaeformis gemeldet. Von dieser Art unterscheidet sich Pleurocerinella srilankai durch den insgesamt weniger bestäubten Thorax (besonders auffällig am vorne unbestäubten Anepisternum), den distal vor den Cerci nicht verschmolzenen Epandrien und dem Fehlen eines unpaaren Zahnes an der Spitze des Aedeagus (Fig. 28).

\section{Diagnosis:}

The identification characteristics of this species are set out in the key below. Pleurocerinella dioctriaeformis is the only other species of Pleurocerinella which occurs within the distribution of Pleurocerinella srilanki. Pleurocerinella srilanki differs from this species in its altogether less-dusted thorax (the undusted anterior anepisternum is especially striking), and in the male by having the epandrium margins fused completely distal to the cerci, and in lacking a conspicuous tooth at the end of the aedeagus (Fig. 28).

\section{Verbreitung:}

Pleurocerinella srilankai ist bislang nur vom Locus typicus auf Sri Lanka bekannt geworden. 


\section{Pleurocerinella tibialis CHEN, 1939}

Chen (1939: 196-197).

Material:

China: 1 + , 17.VIII.1930, „Yati“, coll. CHvÁLA.

Das einzige vorliegende Tier hat dieselben Daten wie der Holotypus, ist aber ein Weibchen. Es handelt sich daher um ein bemerkenswerterweise nicht erwähntes zweites Tier vom Locus typicus („Ya-ti“, China). Der Holotypus befindet sich in der Sammlung der Chinese Academy of Sciences in Beijing (IZCAS) und stand zur Bearbeitung nicht zur Verfügung. Der Beleg von Pleurocerinella tibialis ähnelt im weiblichen Geschlecht Pleurocerinella dioctriaeformis außerordentlich. Da von beiden Arten jeweils nur ein Weibchen vorliegt, kann nicht beurteilt werden, ob die insgesamt schmaler wirkende Theka von Pleurocerinella tibialis ein zuverlässiges Diagnosemerkmal ist. Die männlichen Genitalien von Pleurocerinella tibialis sind unbekannt. Die Flügelfärbung von Pleurocerinella tibialis ist von der von Pleurocerinella dioctriaformis nicht zu unterscheiden. Die in der Originalbeschreibung erwähnte metallische Flügelfärbung von Pleurocerinella tibialis („Ailes pluis ou moins rembrunies avec un reflectt métallique") kann bei dem vorliegenden Tier nicht bestätigt werden. Eventuell liegt das am Alter des Tieres. Trotzdem bleibt fraglich, ob dieses Merkmal zur Unterscheidung von den beiden Arten herangezogen werden kann. Das einzige Merkmal zur Trennung der beiden Arten ist daher die unterschiedliche Bestäubung des Mesoscutums. Außer vom Locus typicus wurde Pleurocerinella tibialis von Zimina (1964) aus „Southern Primorýe (Maritime Territory)“ und von Maeta (1993) aus Japan gemeldet. Aufgrund der dargestellten Bestimmungsschwierigkeiten sollten diese Nachweise aber überprüft werden.

\section{Pleurocerinella violacea KRÖBER, 1930}

(Fig. 20, 22)

KRÖBER (1930: 72-73).

\section{Holotypus:}

$10^{\top}$ aus Indonesien mit folgenden Etiketten: (1) „Type“ [gedruckt, rot]; (2) „Sunda-Exp. Rensch / Rana Mêsé / 20.-21.6.27“ [gedruckt]; (3) „Pleurocerinella / violacea Krb./ det. Kröber 1930“ [erste zwei Zeilen handschriftlich]; „Type“ [rot, gedruckt]. - [Der Typus befindet sich in der Sammlung der Martin Luther Universität, Halle (MLUH) und ist in tadellosem Zustand].

Bislang ist von dieser Art nur das Typenmaterial aus „West Fores, Rana Mêsé“ [Flores, Indonesien] bekannt geworden.

\section{Pleurocerinella spec.}

Es liegen vier Tiere vor, die nicht zugeordnet werden können. Zwei Weibchen aus Kenia (Eastern Prov., Kibwesi Forest, 02²7,90'S, 3754,91'E, Malaise-Falle, 13.-20.XI.1999, leg. R. S. Copeland, coll. Copeland et Stuke) könnten wegen des bestäubten Mesoscutums zu Pleurocerinella copelandi gehören. Aufgrund der Entfernung zum beschriebenen Vorkommen und wegen der Unsicherheit bei der Bestimmung der Weibchen sollen diese Tiere aber zunächst unbestimmt bleiben.

Zwei Männchen aus Südafrika ( $1 \sigma^{\star}$, Cathedral peak, Forestry reserve, Natal, Drakensberg, III.1959, leg. B. R. \& P. J. Stuckenberg, coll. Camras [det. Camras, 1962, als „Pleurocerinella albohalterata“"]) und Tanzania (1 $\mathrm{o}^{\star}$, Dodoma pr., 60km N Kondoa, S04³6', E 3546', $1570 \mathrm{~m}$, 

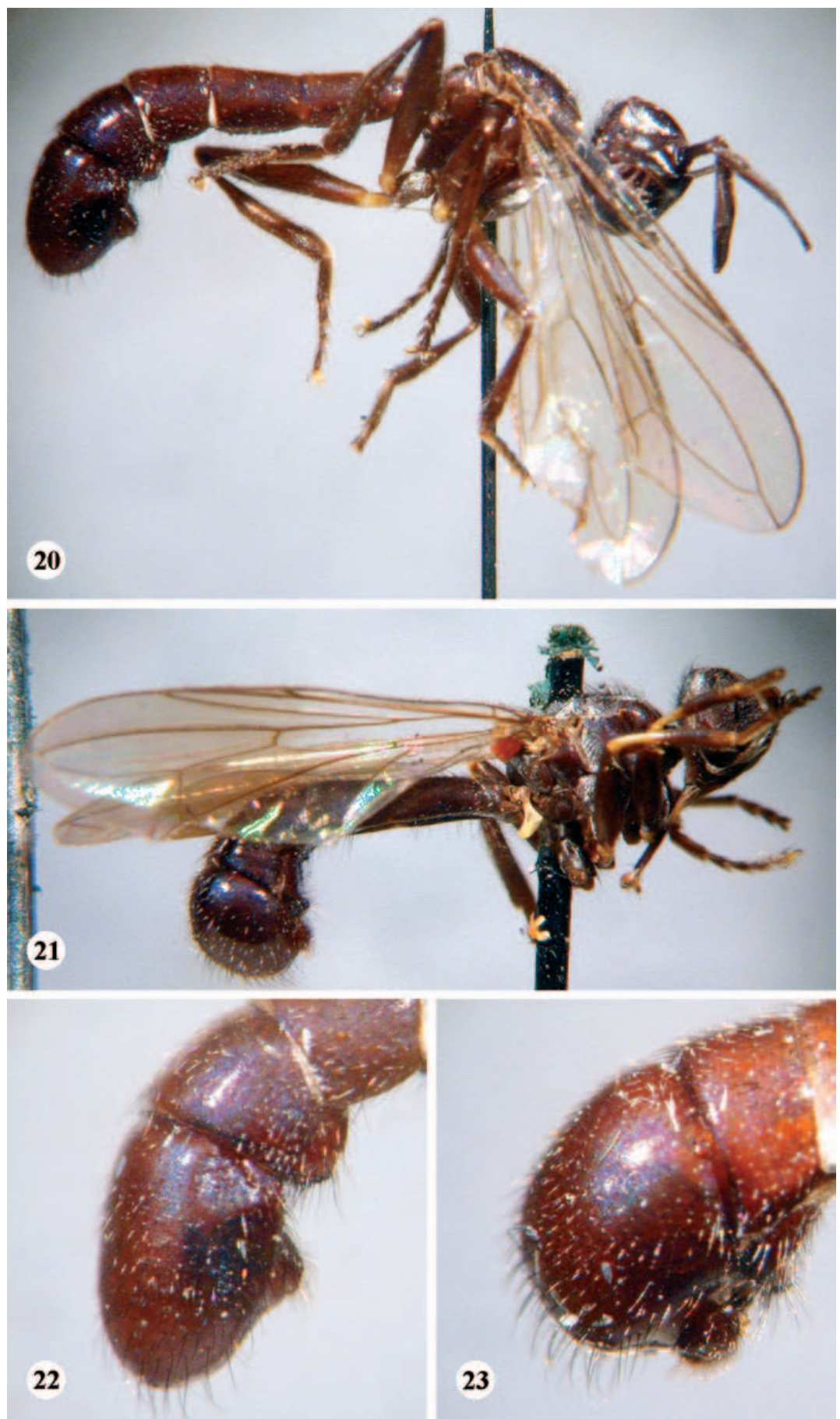

Fig. 20-23: Pleurocerinella. 20 Holotypus Pleurocerinella violacea KRöBER, 1930 ( ( $)$; 21 Lectotypus von Pleurocerinella dioctriaeformis BRUNETTI, 1923 ( $\overbrace{}^{\star}) ; 22$ Abdomenspitze von Pleurocerinella violacea KRöBER, 1930 ( $\left.{ }^{\star}\right)$, Holotypus). 23 Abdomenspitze von Pleurocerinella dioctriaeformis BRUNETTI, 1923 (o von Charbin). 

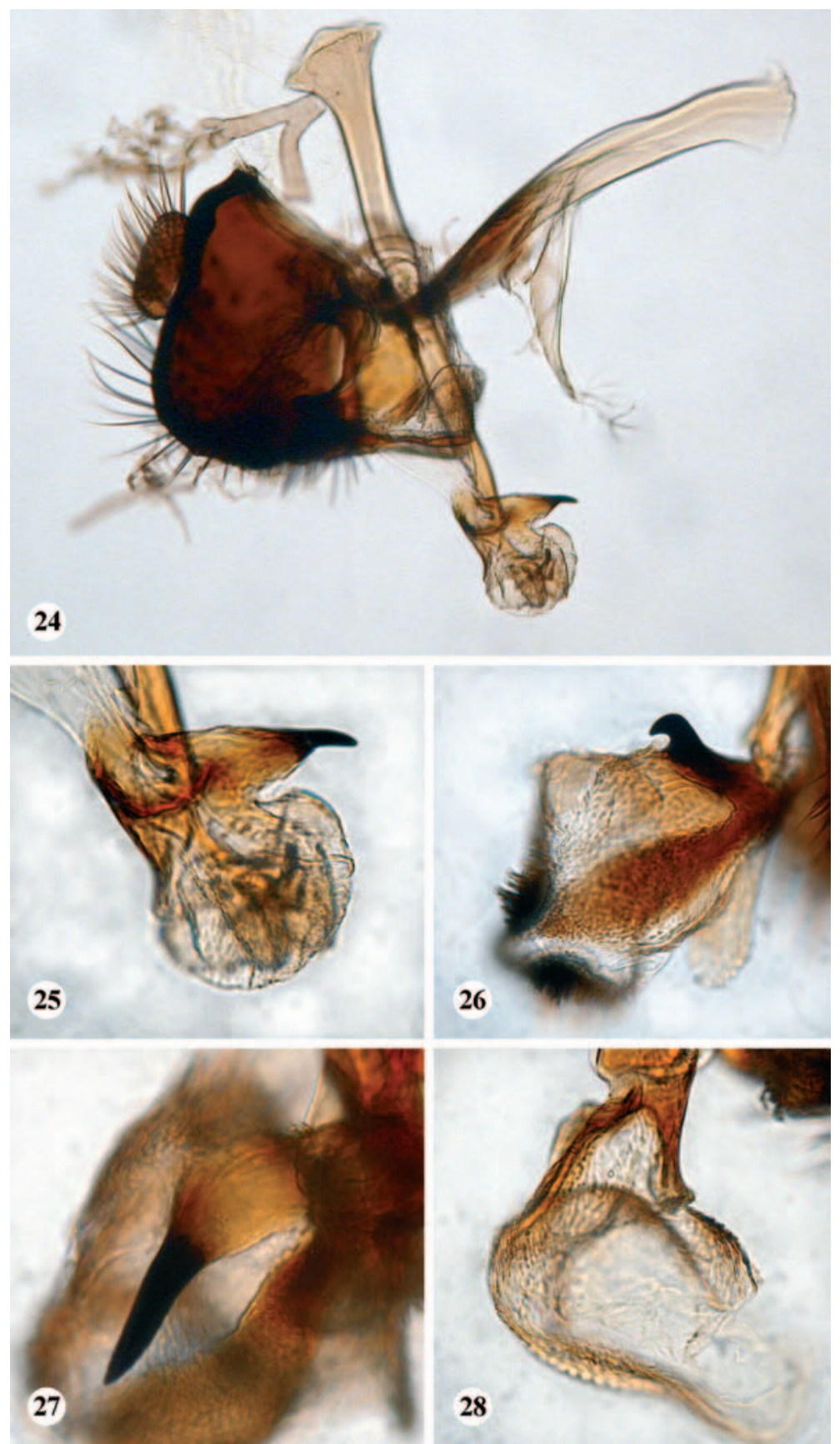

Fig. 24-28: $\sigma^{\star}$-Genitalien von Pleurocerinella: 24 Genital lateral von Pleurocerinella albohalterata Sмiтн, 1960 (Exemplar aus Western Cape); 25 Aedeagusspitze mit unpaarem Zahn von Pleurocerinella albohalterata Sмітн, 1960 (Exemplar aus Western Cape); 26 Aedeagusspitze mit unpaarem Zahn von Pleurocerinella dioctriaeformis BRUNETTI, 1923 (Exemplar aus Godavari, 6000'); 27 unpaarer Zahn von Pleurocerinella haladai spec. nov. (Paratypus vom Locus typicus); 28 Aedeagusspitze ohne unpaaren Zahn von Pleurocerinella srilankai spec. nov. (Holotypus). 


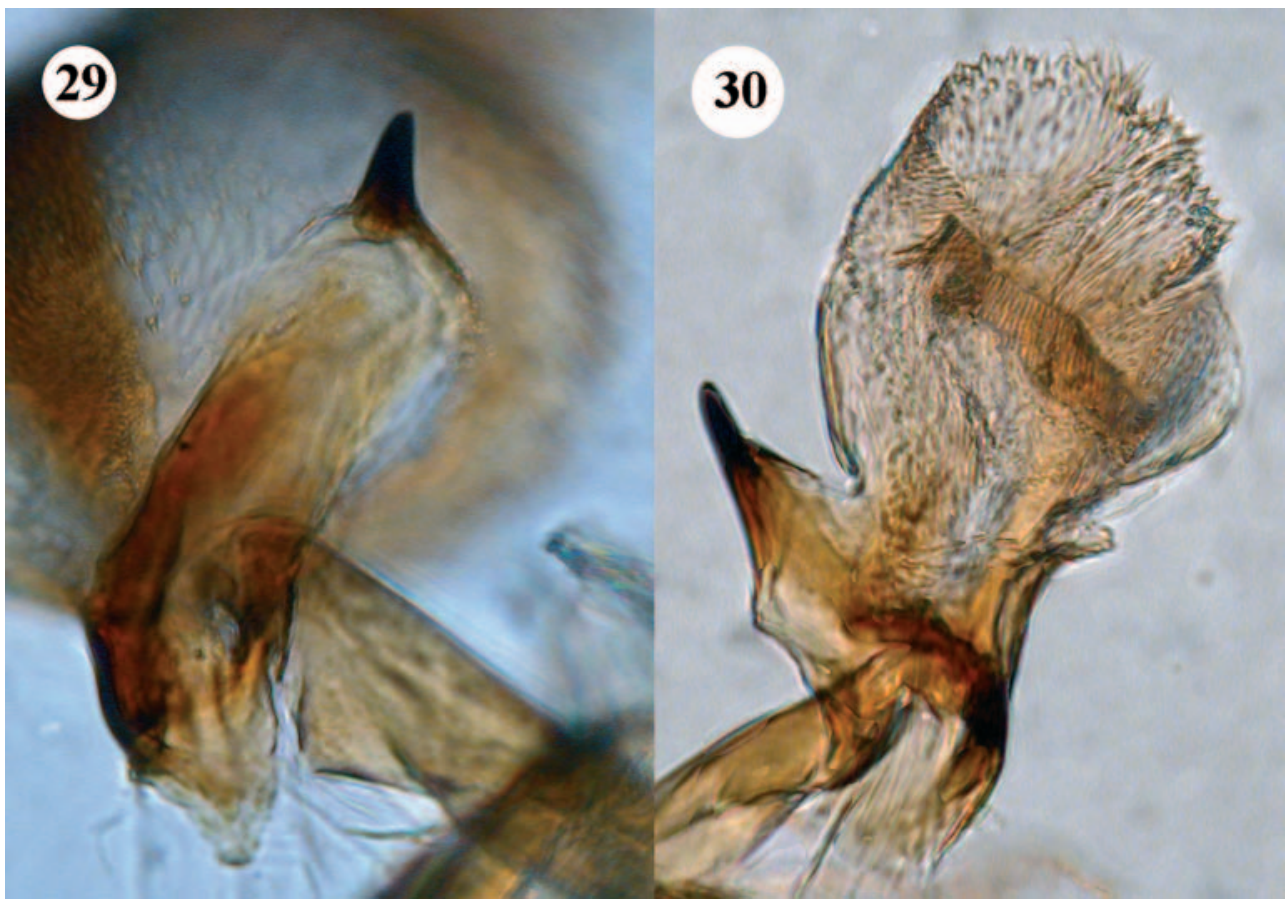

Fig. 29-30: Aedeagusspitze mit unpaarem Zahn von Pleurocerinella: 29 Pleurocerinella bartaki spec. nov.; 30 Pleurocerinella copelandi spec. nov.
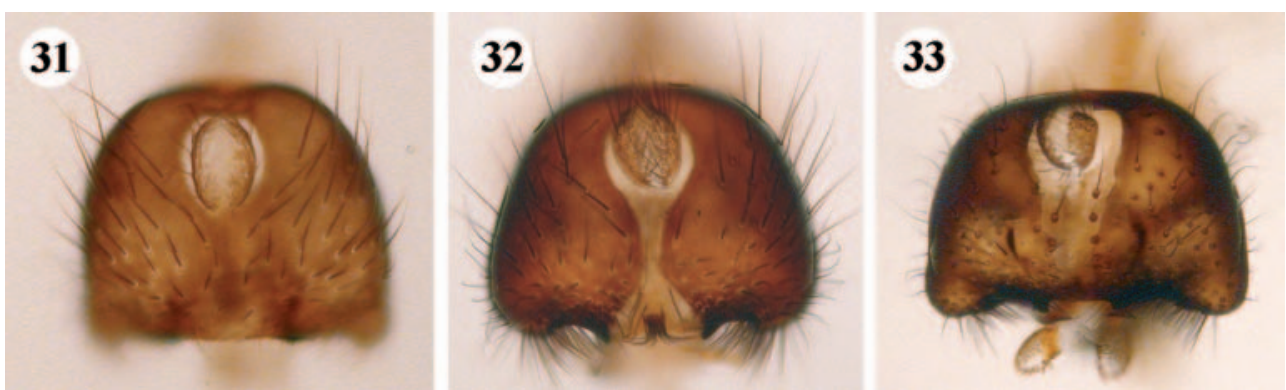

Fig. 31-33: Epandrien mit reduzierten Surstyli verschiedener Pleurocerinella: 31 Pleurocerinella copelandi spec. nov. (Paratypus); 32 Pleurocerinella haladai spec. nov. (Paratypus von Locus typicus); 33 Pleurocerinella bartaki spec. nov. (Holotypus).

19.XII.2006, leg. Halada, coll. Stuke) können nicht zugeordnet werden und gehören vermutlich zu zwei weiteren unbeschriebenen Arten. Die Abgrenzung dieser Arten gegen die benannten Arten ist im Bestimmungsschlüssel dargestellt. Es scheint mir aber notwendig zu sein weiteres Material aus der Gattung Pleurocerinella zu sichten um den Status sicher klären zu können.

\section{Schlüssel für die Arten der Gattung Pleurocerinella Brunetti, 1923}

1 Radialzelle r1 mehr als zur Hälfte ohne Mikrotrichen; alle Femora gleich dunkelbraun bis schwarz; Verbreitung: Paläarktis, Orientalis. 
- $\quad$ Radialzelle r1 meistens ganz oder zumindest überwiegend mit Mikrotrichen; Vorder- und Mittelfemur orange oder deutlich heller als der Hinterfemur; Verbreitung: Afrotropis. .... 5

2 Anepisternum im vorderen Teil unbestäubt, daran ein dichter Bestäubungsstreifen anschließend (Fig. 18); $0^{\star}$-Genital: Epandrien distal vor den Cerci vollständig verschmolzen, unpaarer Zahn an Aedeagusspitze fehlt (Fig. 28).

srilankai Stuke \& CAmras spec. nov.

- $\quad$ Anepisternum entweder vollständig bestäubt oder ohne Bestäubungsstreifen; ${ }^{\star}$-Genital: Epandrien distal vor den Cerci getrennt (Merkmalszustand von tibialis unbekannt); unpaarer Zahn an Aedeagusspitze vorhanden (nur von dioctriaeformis bekannt). 3

3 Anepisternum, Postpronotum (Humeri), Seiten des Mesoscutums und Stirn unbestäubt; Flügel apikal nicht verdunkelt; Hinterleibspitze des Männchens (Tergit 6) weit ausgezogen (Fig. 22); هึ-Genital unbekannt. violaceae KRÖBER, 1930

- $\quad$ Anepisternum, Postpronotum (Humeri), Seiten des Mesoscutums und obere Stirn deutlich bestäubt; Flügel apikal undeutlich verdunkelt; Hinterleibspitze des Männchens von dioctriaeformis (Tergit 6) nicht ausgezogen (Fig. 23), ơ von tibialis unbekannt.

4 Mesoscutum nur lateral bestäubt, medial deutlich abgesetzt unbestäubt; $0^{\star}$-Genital: der unpaarer Zahn an Aedeagusspitze entsprechend Fig. 26.

dioctriaeformis BRUNETTI, 1923

Mesoscutum vollständig bestäubt; ơ-Genital unbekannt.

tibialis Chen, 1939

5 Flügel vollständig mit Mikrotrichen besetzt; Femora und Tibien orange; Hinterfemur ohne schwarze Borsten auf der Unterseite; Mesoscutum lateral unbestäubt; ${ }^{\top}{ }^{\star}$-Genitalien: Epandrien distal vor den Cerci verschmolzen; unpaarer Zahn an Aedeagusspitze entsprechend Fig. 25. albohalterata SмIтH, 1960

- $\quad$ Flügelzelle bm und br basal ohne Mikrotrichen; zumindest Hinterfemur schwarzbraun bis hellbraun; Hinterfemur mit oder ohne schwarzen Borsten auf der Unterseite; Mesoscutum lateral bestäubt; $\sigma^{\star}$-Genitalien: Epandrien distal vor den Cerci getrennt oder verschmolzen.

6 Mesoscutum in der Mitte und lateral deutlich bestäubt, submedial glänzend; unpaarer Zahn an Aedeagusspitze entsprechend Fig. 30. copelandi STUKE spec. nov.

Mesoscutum nur lateral bestäubt, medial glänzend. 7

Von den folgenden Arten können nur die Männchen bestimmt werden, da von den Weibchen bislang nicht genug Material vorliegt.

7 Epandrien distal vor den Cerci vollständig getrennt (Fig. 32); unpaarer Zahn an Aedeagusspitze entsprechend Figur 27: sehr langgestreckt. haladai STUKe spec. nov.

- $\quad$ Epandrien distal vor den Cerci verschmolzen (Fig. 33); unpaarer Zahn an Aedeagusspitze kürzer (Fig. 29). 8

$8 \quad$ Hinterfemur auf der Unterseite mit schwarzen Borsten; Basalzellen br und bm fast vollständig ohne Mikrotrichen; unpaarer Zahn an Aedeagusspitze entsprechend Fig. 29.

bartaki STUKE spec. nov.

- $\quad$ Hinterfemur auf der Unterseite ohne schwarze Borsten; Basalzellen br und bm nur basal ohne Mikrotrichen. spec. 1 , spec. 2 
Key to the species of the genus Pleurocerinella Brunetti, 1923

1 Wing cell $\mathrm{r} 1$ with at least the basal half lacking microtrichia; all femora uniformly black or dark brown; distribution: Palaearctic and Oriental regions. 2

Wing cell $\mathrm{r} 1$ completely or mostly covered with microtrichia; fore and middle femora orange brown or clearly paler than the hind femur; distribution: Afrotropical region.

2 Anterior anepisternum shining, with a dense dusted stripe posteriorly (Fig. 18); $\sigma^{\star}$-genitalia: epandrium margins fused completely distal to the cerci; lacks a robust single tooth at the tip of the aedeagus (Fig. 28). srilankai Stuke \& Camras spec. nov.

- Anepisternum completely dusted, or if shining, then without a dense dusted stripe; $\sigma^{\star}-$ genitalia: epandrium margins separated distal to the cerci (character state remains unknown in tibialis); may have an obvious robust single tooth at the tip of the aedeagus (at present, only known in dioctriaeformis). 3

3 Anepisternum, postpronotum, edges of the mesoscutum and upper part of the frons shining; wing apex not clearly brownish-tinged; last segment of the male abdomen (tergite 6) elongated (Fig. 22); structure of the aedeagus remains unknown. violaceae KRÖвER, 1930 Anepisternum, postpronotum, edges of the mesoscutum and upper part of the frons obviously dusted; wing apex clearly brownish tinged; last segment of the male abdomen (tergite 6) of dioctriaeformis not elongated (Fig. 23), ơ of tibialis remains unknown. 4

4 Mesoscutum only dusted laterally, shining medially; $\sigma^{\star}$-genitalia: single robust tooth at the tip of the aedeagus as in Fig. 26. dioctriaeformis BRUNETTI, 1923

- Mesoscutum completely dusted; $\sigma^{\top}$-genitalia remains unknown. tibialis CHeN, 1939

$5 \quad$ Wing completely covered with microtrichia; femora and tibiae orange; hind femur lacking black spines ventrally; mesoscutum shining laterally; $o^{x}$-genitalia: epandrium margins fused completely distal to the cerci; tooth at the tip of the aedeagus as in Figur 25.

albohalterata SMITH, 1960

Wing cells bm and br lacking microtrichia basally; at least hind femur dark-brown to lightbrown; hind femur with or without black spines ventrally; mesoscutum dusted laterally; $\sigma^{\star}$-genitalia: epandrium margins separated or fused distal to the cerci. 6

6 Mesoscutum dusted laterally and medially, submedially shining; tooth at the tip of the aedeagus as in Fig. 30. copelandi STUKE spec. nov.

- Mesoscutum only dusted laterally, medially shining. 7

The following species can only currently be identified in the male, due to the lack of available female material.

$7 \quad$ Epandrium margins separated distal to the cerci (Fig. 32); tooth at the tip of the aedeagus as in Fig. 27: tooth elongated. haladai STUKe spec. nov.

- $\quad$ Epandrium margins fused distal to the cerci (Fig. 33); tooth at the tip of the aedeagus as in Fig. 29: tooth shorter. 8

$8 \quad$ Hind femur with black spines ventrally; wing cells br and bm more or less completely lacking microtrichia; tooth at the tip of the aedeagus as in Fig. 29. 
- $\quad$ Hind femur without black spines ventrally; wing cells br and bm only lacking microtrichia at base. spec. 1, spec. 2

\section{Danksagung}

Für das Überlassen oder das Ausleihen von Material aus ihren oder von ihnen betreuten Sammlungen danke ich Prof. Dr. Miroslav Bartak (Prag), Prof. Dr. Milan Chvála (Prag); Sid Camras (Chicago), Robert S. Copeland (Nairobi), Dr. Martin Hauser (Sacramento), Dr. Ashley H. Kirk-Spriggs (Grahamstown), Dr. Libor Mazánek (Olomouc), Dr. Frank Menzel (Müncheberg), Dr. Karla Schneider (Halle), Dr. Andreas Stark (Halle), Dr. Nigel Wyatt (London) und Dr. Joachim Ziegler (Berlin). Rebekah Shuman (Chicago) half bei der elektronischen Kommunikation mit Sid Camras. David Clements (Cardiff) prüfte geduldig die englischsprachigen Teile verschiedener Manuskriptversionen.

\section{Literatur}

Brunetri, E. 1923: Pipunculidae, Syrphidae, Conopidae, Oestridae. - Fauna of British India 3: xi + $1-424$.

Brunetti, E. 1925: New Afrikan Conopidae. - Annals and Magazine of Natural History 9: 101-112.

Camras, S. 1962: Records and descriptions of African Conopidae (Diptera). - Revue de Zoologie et de Botanique Africaines 66: 203-242.

Camras, S. 2000: New information on Afrotropical Conopidae (Diptera). - Entomologist's Monthly Magazine 136: 219-233.

Chen, S. H. 1939: Étude sur les Diptères Conopides de la Chine. - Notes d' Entomologie Chinoise 6: 161-231.

KröвеR, O. 1930: Die Dipterenausbeute der Sunda-Expedition Rensch. Beitrag II: Therevidae und Conopidae. - Zoologischer Anzeiger 89: 65-73.

Maeta, Y. \& MacFarlane, R. P. 1993: Japanese Conopidae (Diptera): Their Biology, Overall Distribution, and Role as Parasites of Bumble Bees (Hymenoptera, Apidae). - Japanese Journal of Entomology 61: 493-509.

McAlpine, J. F. 1981: Morphology and Terminology - Adults. - In: McAlpine, J. F. et al. (eds.): Manual of Nearctic Diptera. Volume 1; Ontario: Research Branch Agriculture Canada, Monograph No. 27: 9-63.

Smith, K. G. V. 1960: A new species of Pleurocerinella Brunetri (Dipt., Conopidae) from south Africa. - Entomologist's Monthly Magazine 96: 43-44.

Sмith, K. G. V. 1980: 39. Family Conopidae. - In: Crosskey, R. W. (eds): Catalogue of the Diptera of the Afrotropical Region; London: British Museum (Natural History): 511-517.

StUckenberg, B. R. 1999: Antennal evolution in the Brachycera (Diptera), with a reassessment of terminology relating to the flagellum. - Studia dipterologica 6: 33-48.

Stuke, J.-H. 2008: Two new genera of Conopidae from the Afrotropical and Neotropical Region (Diptera). - Zootaxa 1874: 50-56.

Zimina, L. V. 1964: Some interesting Diptera (Conopidae and Syrphidae) from the Far East. - Entomological Review 43: 236-238.

\section{Anschrift des Verfassers:}

Dr. Jens-Hermann Stuke

Roter Weg 22

26789 Leer

Deutschland

E-Mail: jstuke@zfn.uni-bremen.de
Subject editor:

Dr. F. Menzel 